\title{
Time-resolved fluorescence study of excitation energy transfer in the cyanobacterium Anabaena PCC 7120
}

\author{
Parveen Akhtar ${ }^{1,2} \cdot$ Avratanu Biswas $^{1,3,4} \cdot$ Nia Petrova $^{5} \cdot$ Tomas Zakar $^{1} \cdot$ Ivo H. M. van Stokkum $^{4} \cdot$ Petar H. Lambrev $^{1}$ (D
}

Received: 28 December 2019 / Accepted: 7 February 2020 / Published online: 19 February 2020

(c) The Author(s) 2020

\begin{abstract}
Excitation energy transfer (EET) and trapping in Anabaena variabilis (PCC 7120) intact cells, isolated phycobilisomes (PBS) and photosystem I (PSI) complexes have been studied by picosecond time-resolved fluorescence spectroscopy at room temperature. Global analysis of the time-resolved fluorescence kinetics revealed two lifetimes of spectral equilibration in the isolated PBS, 30-35 ps and 110-130 ps, assigned primarily to energy transfer within the rods and between the rods and the allophycocyanin core, respectively. An additional intrinsic kinetic component with a lifetime of 500-700 ps was found, representing non-radiative decay or energy transfer in the core. Isolated tetrameric PSI complexes exhibited biexponential fluorescence decay kinetics with lifetimes of about $10 \mathrm{ps}$ and $40 \mathrm{ps}$, representing equilibration between the bulk antenna chlorophylls with low-energy "red" states and trapping of the equilibrated excitations, respectively. The cascade of EET in the PBS and in PSI could be resolved in intact filaments as well. Virtually all energy absorbed by the PBS was transferred to the photosystems on a timescale of $180-190$ ps.
\end{abstract}

Keywords Allophycocyanin $\cdot$ Light harvesting $\cdot$ Photosynthesis $\cdot$ Photosystem I $\cdot$ Phycobilisomes $\cdot$ Phycocyanin

The authors would like to dedicate this work to the memory of Dr. Zoltán Gombos.

Electronic supplementary material The online version of this article (https://doi.org/10.1007/s11120-020-00719-w) contains supplementary material, which is available to authorized users.

Petar H. Lambrev

lambrev.petar@brc.hu

1 Biological Research Centre, Szeged, Temesvári krt. 62, Szeged 6726, Hungary

2 ELI-ALPS, ELI-HU Nonprofit Ltd., Wolfgang Sandner u. 3, Szeged 6728, Hungary

3 Doctoral School of Biology, University of Szeged, Közép Fasor 52, Szeged 6726, Hungary

4 Department of Physics and Astronomy and LaserLaB, Faculty of Science, Vrije Universiteit Amsterdam, De Boelelaan 1081, 1081 HV Amsterdam, The Netherlands

5 Institute of Biophysics and Biomedical Engineering, Bulgarian Academy of Sciences, Acad G. Bontchev Str., B1. 21, 1113 Sofia, Bulgaria

\section{Introduction}

Light harvesting in cyanobacteria relies on phycobilisomes (PBS) - the large multimeric water-soluble assemblies of phycobiliproteins (PBPs) and linker proteins that efficiently absorb light in the spectral range between 550 and $650 \mathrm{~nm}$ (Harris et al. 2018; Bar-Eyal et al. 2018). PBS are commonly described as hemidiscoidal structures consisting of PBP trimers $(\alpha \beta)_{3}$ and hexamers $(\alpha \beta)_{6}$ assembled together with linker proteins into a central core of allophycocyanin (APC) with radiating rods containing phycocyanin (PC) and in some species phycoerythrin (PE) or phycoerythrocyanin (PEC) (Arteni et al. 2009; MacColl 2004). There are, however, large variations in the PBS organization and composition and deviations from this fundamental design depending on the species and growth conditions. Absorbed energy is transferred to the photosystems with a very high quantum efficiency (Scott et al. 2006; Glazer 1984)—PBS are generally considered as antenna for photosystem II (PSII) but they can also supply excitation energy to photosystem I (PSI), either indirectly via "spillover" from PSII or directly (Mullineaux 1994; Liu et al. 2013; Chukhutsina et al. 2015).

Excitation energy transfer (EET) has been studied in isolated PBPs (Holzwarth et al. 1990; Debreczeny et al. 1993; 
Switalski and Sauer 1984; Choubeh et al. 2018), PBS substructures (Sandström et al. 1988; Zhao et al. 1998), intact PBS (Tian et al. 2012; Nganou et al. 2016; Zhang et al. 1997; van Stokkum et al. 2018) as well as in whole cells of various species (Mullineaux and Holzwarth 1991; Tian et al. 2011; Acuña et al. 2018a, b; Chukhutsina et al. 2015), including Anabaena variabilis (Bittersmann et al. 1988; Nultsch et al. 1990). Despite the abundance of experimental data and models, there are contradicting views about the kinetics and ratelimiting steps of EET in the PBS and between them and the photosystems-partly because of the sheer complexity of the PBS. Individual PBP-PC or APC—contain phycobilin (PCB) pigments with different spectral properties and EET between them occurs on timescales from hundreds of femtoseconds to several tens of picoseconds in isolated $(\alpha \beta)_{3}$ trimers (Choubeh et al. 2018; Debreczeny et al. 1993; Zhao et al. 1998; Holzwarth et al. 1990). Association of the trimers into hexamers accelerates the equilibration because of more parallel contacts between the pigments and EET along the PBS rods (Holzwarth 1991) can be faster than equilibration within the individual subunits (Suter and Holzwarth 1987). The overall equilibration between the rods and the APC core is found to be in the order of 20 to $120 \mathrm{ps}$, depending on the size and number of rods (Holzwarth 1991; Tian et al. 2012). EET between the PBS and the photosystems has been proposed to be either very slow (Mullineaux and Holzwarth 1991) or very fast (Tian et al. 2011) and equilibration within the assembled core is not well defined. An elaborated functional compartmental model of EET in Synechocystis PCC 6803 has been recently reported (van Stokkum et al. 2018), according to which the slowest EET rates are between the core cylinders (115-145 ps) and between the rods and the core (68-115 ps). By time-resolved fluorescence, Acuña et al. (2018b) reported a 20 ps EET time from the PBS terminal emitter to PSII, i.e. faster than the EET within the PBS.

The PBS of the nitrogen-fixating cyanobacterium Anabaena PCC 7120 (hereafter called Anabaena) belong to the same hemidiscoidal family, as for instance the well-studied Synechocystis 6803, but are different in several ways (Ducret et al. 1998; Glauser et al. 1992). They contain up to eight rods (compared to six in Synechocystis), composed of not only PC but also PEC hexamers absorbing with a maximum at $575 \mathrm{~nm}$ owing to the bound phycoviolobilin chromophore (PVB). The penta-cylindrical APC core contains two supplementary flanking cylinders in addition to the two basal and the top cylinder found in the tri-cylindrical core of Synechocystis. Anabaena has unusual tetrameric organization of PSI (Watanabe et al. 2014), whose structure has been recently revealed by cryoelectron microscopy (Kato et al. 2019). The so-called "red" chlorophylls (Chls) in PSI absorbing light at longer wavelengths than the reaction centre (RC) (Gobets et al. 2001; El-Mohsnawy et al. 2010; Karapetyan et al.
2006) are especially prominent in Anabaena, which shifts the fluorescence emission maximum by $8 \mathrm{~nm}$ compared to Synechocystis. Supercomplexes containing tetrameric PSI and a special type of PBS containing only rods of PEC and $\mathrm{PC}$ and the rod-core linker CpcL have been observed in vegetative and nitrogen-fixating Anabaena cells (Watanabe et al. 2014). The CpcL-PBS is thought to be a specific antenna for PSI (Kondo et al. 2007; Liu et al. 2019; Niedzwiedzki et al. 2019); however, it is not yet studied in detail. The specific characteristics of the PBS and PSI in Anabaena are expected to affect EET in the complexes and consequently in the cells.

In this work, we applied steady-state and time-resolved fluorescence spectroscopy to study the dynamics of EET in isolated PBS, PSI and intact filaments of Anabaena PCC 7120. We show that spectral equilibration in the PBS occurs on timescales up to $125 \mathrm{ps}$ and is a rate-limiting factor in the kinetics of intact cells where most excitations on the PBS are trapped on a timescale of about $180 \mathrm{ps,} \mathrm{i.e.} \mathrm{slower}$ than the charge separation in either PSI or PSII. We further resolved the kinetics of exciton equilibration and trapping in tetrameric PSI both in vivo and in the isolated complex.

\section{Materials and methods}

\section{Sample preparation}

\section{Cell growth conditions}

Vegetative cells of Anabaena variabilis (PCC 7120) were cultivated in BG-11 (Rippka et al. 1979) medium supplemented with $5 \mathrm{mM}$ HEPES-NaOH $(\mathrm{pH} 7.5)$ at $30^{\circ} \mathrm{C}$ under continuous white light illumination at an intensity of $40 \mu \mathrm{mol}$ photons $\mathrm{m}^{-2} \mathrm{~s}^{-1}$. Cultures were aerated on a gyratory shaker operating at $100 \mathrm{rpm}$.

\section{Isolation of PBS}

PBS were prepared from Anabaena filaments according to Garnier et al. (1994) with some adjustments. Briefly, photoautotrophically grown cells were centrifuged to pellet at $7000 \times g$ at $25{ }^{\circ} \mathrm{C}$. The pellet was resuspended in $0.75 \mathrm{M}$ phosphate buffer, $1 \mathrm{mM}$ benzamidine hydrochloride hydrate, $1 \mathrm{mM}$ ethylenediaminetetraacetic acid ( $\mathrm{pH} 7.0)$, and $1 \mathrm{mM}$ of phenylmethylsulfonyl fluoride and homogenized by using a bead-beater homogenizer followed by centrifugation at $3000 \times g$ for $5 \mathrm{~min}$ at $14{ }^{\circ} \mathrm{C}$ to remove the filament debris. The supernatant was then treated with 3\% Triton-X100 with continuous stirring for $45 \mathrm{~min}$ at room temperature in dark and centrifuged at $70,000 \times g$ for $30 \mathrm{~min}$ to remove the unsolubilized material. The appropriate sample fraction was collected and loaded onto a sucrose density step gradient (1 M, $0.75 \mathrm{M}, 0.5 \mathrm{M}, 0.25 \mathrm{M}$ sucrose) and centrifuged for $16 \mathrm{~h}$ at 
$26,000 \mathrm{rpm}, 14{ }^{\circ} \mathrm{C}$ for further purification. The gradient fraction containing PBS was then collected and characterized by steady-state spectroscopy for further use.

\section{Isolation of photosystem I}

PSI was isolated from the 1-week-old cells according to Vajravel et al. (2017). Briefly, thylakoid membranes were isolated in a medium containing $25 \%$ glycerol, $0.5 \mathrm{mM}$ phenylmethanesulfonyl fluoride and $1 \mathrm{mM}$ benzamidine by breaking the cells by glass beads in a beater. Isolated thylakoid membranes were further solubilized with $2 \% n$-dodecyl $\beta$-D-maltoside ( $\beta$-DDM) and loaded on a stepwise (6 steps, 0.2-0.9 M) sucrose gradient containing $20 \mathrm{mM}$ HEPES (pH 7) and $0.05 \%$ of $\beta$-DDM followed by centrifugation at $220,000 \times g$ for $14 \mathrm{~h}$ at $4{ }^{\circ} \mathrm{C}$. PSI-containing fractions of the gradient were collected by a syringe. The sample was washed in a medium containing $0.03 \% \beta$-DDM and concentrated using Amicon Ultra filters (Millipore), characterized and frozen in liquid $\mathrm{N}_{2}$ and stored at $-80{ }^{\circ} \mathrm{C}$ until use. The purity of the samples was confirmed by SDS-PAGE (Supplementary Fig. S1).

\section{Absorption and fluorescence spectroscopy}

Absorption spectra were recorded between 350 and $750 \mathrm{~nm}$ at room temperature using an Evolution 500 dual-beam spectrophotometer (Thermo Scientific). Samples were diluted with their corresponding media to an absorbance of one at the red maximum. Measurements were performed in a standard glass cell of $1 \mathrm{~cm}$ optical path length.

Fluorescence emission spectra at $77 \mathrm{~K}$ were recorded with a Fluorolog 3 double-monochromator spectrofluorometer (Horiba Jobin-Yvon, USA). In the case of isolated samples, $40-50 \mu \mathrm{l}$ of solution with absorbance of 0.3 was evenly placed onto a Whatman GF/C glass microfiber filter and immersed in liquid nitrogen in a Dewar glass vessel. For measuring fluorescence from intact cells, a culture volume containing $5 \mu \mathrm{g}$ Chl was filtered onto a Whatman GF/C filter. Emission spectra were recorded in the range of $620-800 \mathrm{~nm}$ with excitation at 460 or $580 \mathrm{~nm}$ and spectral bandwidth of 5 and $2 \mathrm{~nm}$ for excitation and emission, respectively.

\section{Time-resolved fluorescence}

Time-correlated single-photon counting (TCSPC) measurements at room temperature were done using a FluoTime 200/ PicoHarp 300 spectrometer (PicoQuant, Germany) equipped with a microchannel plate detector (R3809U, Hamamatsu, Japan) as described in Akhtar et al. (2016). Excitation pulses at 460 or $580 \mathrm{~nm}$ at $20 \mathrm{MHz}$ repetition rate, $\sim 0.1$ pJ energy, were obtained from a Fianium WhiteLase Micro (NKT Photonics, UK) supercontinuum laser coupled to a monochromator. The total instrument response (IRF) width was $48-50 \mathrm{ps}$, measured using $1 \%$ Ludox as scattering solution. Isolated PBS and PSI complexes were diluted to an absorbance of 0.03 at the excitation wavelength and intact filaments to 0.05 at $750 \mathrm{~nm}$ and circulated through a $1.5-\mathrm{mm}$ pathlength flow cell. The fluorescence decays were recorded at emission wavelengths between 600 and $800 \mathrm{~nm}$ with 6 or $8 \mathrm{~nm}$ step to construct time-resolved emission spectra. Global multiexponential lifetime analysis with IRF reconvolution was performed using MATLAB.

To keep the PSII RC from being closed by the excitation beam, the cell suspension was circulated at a flow rate of $4 \mathrm{ml} / \mathrm{min}$. Alternatively, measurements with closed PSII RCs were performed by adding $20 \mu \mathrm{M}$ DCMU to the medium, additional background light and lowering the flow rate $\sim$ tenfold.

\section{Results}

\section{Absorption and fluorescence spectra}

PBS and PSI complexes isolated from Anabaena were characterized by steady-state absorption and fluorescence spectroscopy (Fig. 1). The absorption spectrum of PBS is dominated by a broad band with a maximum at $620 \mathrm{~nm}$, corresponding to absorption of PC and shoulders on the blue and red side of the maximum. Gaussian decomposition analysis revealed additional bands at $573 \mathrm{~nm}$, originating from PEC, $638 \mathrm{~nm}$ - from red-shifted PC, and $656 \mathrm{~nm}$, corresponding to APC. Overall, the absorption spectrum is very similar to those previously published for Anabaena (Jallet et al. 2014). The fluorescence emission spectrum (Fig. 1b) at $77 \mathrm{~K}$ has a maximum at $685 \mathrm{~nm}$ reflecting the terminal emitter APC pigments. Additionally, several bands with relatively small amplitudes are observed on the blue side and a broad vibronic tail extends to the near-infrared region. The spectrum is also broadly similar to the earlier reported, only slightly more structured (Jallet et al. 2014), allowing us to verify the peak positions by Gaussian decomposition. The analysis shows the presence of two spectral forms of APC, emitting at 664 and $685 \mathrm{~nm}$, PEC emitting around $617 \mathrm{~nm}$ and two forms of PC at 640 and $650 \mathrm{~nm}$. It must be noted though that the Gaussian fit components of both the absorption and emission spectra neither represent all spectral forms in the complex nor their actual bandwidths or stoichiometric ratios. For example, the broader absorption band at $553 \mathrm{~nm}$ and emission band at $685 \mathrm{~nm}$ likely cover multiple spectral forms. The longest-wavelength emission component may be due to vibrational overtones. Because excitation energy is efficiently transferred to the terminal emitters $(685 \mathrm{~nm})$, all other bands have relatively low intensity $(<20 \%$ of the main maximum), despite the high pigment abundance. The 
Fig. 1 Steady-state absorption and fluorescence spectra of isolated PBS from Anabaena PCC 7120 and Gaussian decomposition analysis. a Absorption spectrum at room temperature and best-fit Gaussian components centred at $553,573,617$, 639, $657 \mathrm{~nm}$. b Fluorescence emission spectrum recorded at $77 \mathrm{~K}$ (excitation $580 \mathrm{~nm}$ ) and best-fit Gaussian components centred at $617,641,651,664$, $685,686,710 \mathrm{~nm}$
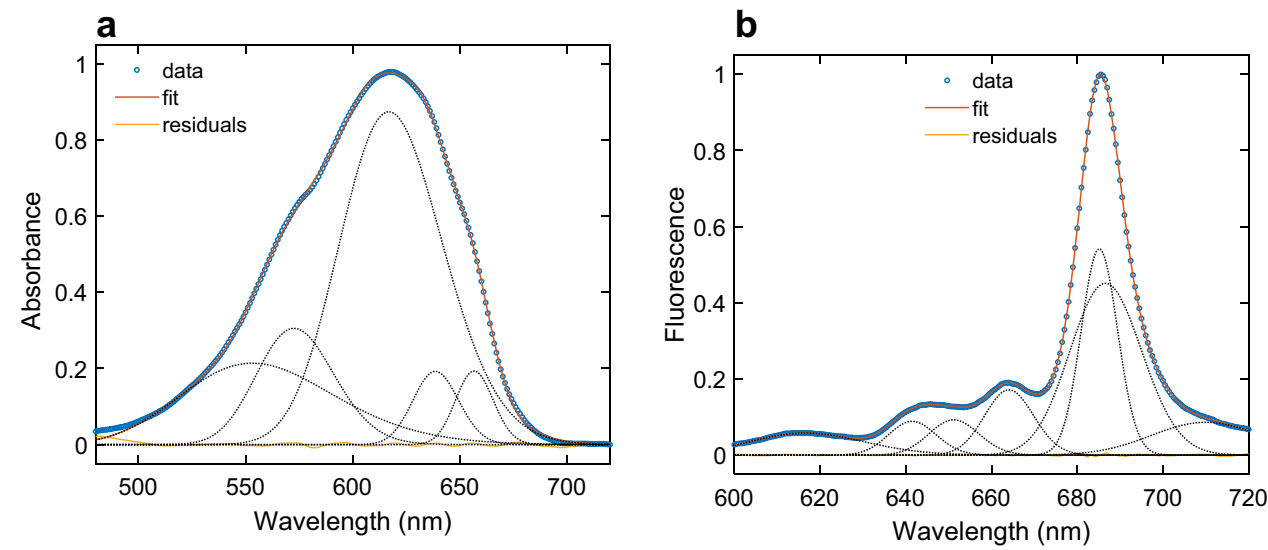

emission maximum of isolated PBS at room temperature is observed around $670 \mathrm{~nm}$ and the spectrum is broader and less featured than at $77 \mathrm{~K}$ (Supplementary Fig. S2a).

The absorption and fluorescence spectra of PSI isolated by sucrose gradient ultracentrifugation are shown in Fig. 2. The absorption spectrum is generally similar to the spectrum of PSI isolated from other cyanobacteria (Andrizhiyevskaya et al. 2002; Lundell et al. 1985; Rögner et al. 1990). The main absorption bands correspond to Chl $a$ Soret and $Q$ transitions and the peak at $500 \mathrm{~nm}$ originates from $\beta$-carotene. The absorption spectrum extends well beyond $700 \mathrm{~nm}$ owing to the presence of low-energy "red" Chls, the number of which varies among different cyanobacterial species (Gobets et al. 2001; Karapetyan et al. 2006). The $77 \mathrm{~K}$ fluorescence emission spectrum (Fig. 2b) is very similar to the recently reported spectrum of PSI tetramers from Anabaena (Kato et al. 2019). As excitation energy is trapped by the red Chls at low temperature, the emission maximum is at $725 \mathrm{~nm}$ and the intensity at wavelengths shorter than $700 \mathrm{~nm}$ is negligible - in contrast, the fluorescence maximum at room temperature is at $684 \mathrm{~nm}$ (Supplementary Fig. S2b).

The absorption spectrum of intact Anabaena cells and the fluorescence emission spectrum recorded at $77 \mathrm{~K}$ (Fig. 3) are similar to those previously published for the same species (Ogawa et al. 1970; Peterson et al. 1981). Between the Soret and $Q_{\mathrm{y}}$ absorption bands of Chl $a$, at 440 and $680 \mathrm{~nm}$, respectively, the PBS absorption peaking at $628 \mathrm{~nm}$ is visible (Fig. 3a). For fluorescence emission spectra either $590 \mathrm{~nm}$ light was used, exciting mainly PC, or $460 \mathrm{~nm}$ light, exciting mainly Chls in PSI and PSII. The $77 \mathrm{~K}$ fluorescence spectrum excited at $460 \mathrm{~nm}$ has a maximum at $730 \mathrm{~nm}$ showing that most of the emission originates from the red Chls in PSI (Fig. 3b). The peak around $693 \mathrm{~nm}$ that can be attributed to PSII has much lower intensity, because of the relative abundance of PSI Chls. The emission spectrum with $580 \mathrm{~nm}$ excitation has well-defined peaks at 649, 660, 692 and $725 \mathrm{~nm}$. The emission peaks at 649 and $660 \mathrm{~nm}$ correspond to PC and APC, $692 \mathrm{~nm}$ to PSII and $725 \mathrm{~nm}$ to PSI emission. The areas under the PSI and PSII peaks are comparable, indicating that PBS transfers to both PSI and PSII with similar efficiency, assuming that the fluorescence quantum yield is similar for both bands.

Qualitatively similar dependence of the emission spectra on excitation wavelength is observed at room temperature; however, the spectra are generally blue-shifted and less featured (Supplementary Fig. S3). The strong excitation
Fig. 2 Absorption and fluorescence spectra of isolated tetrameric PSI form Anabaena. a Absorption spectrum at room temperature. b Fluorescence emission spectrum recorded at $77 \mathrm{~K}$ (excitation $460 \mathrm{~nm}$ )
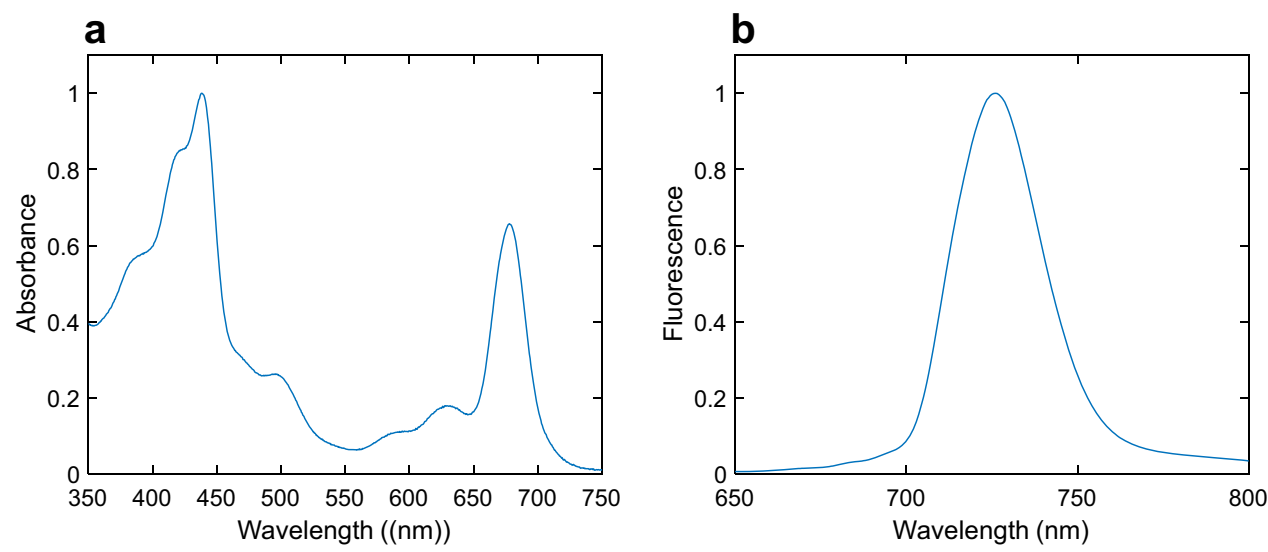
Fig. 3 Absorption and fluorescence spectra of intact cells. a Absorption spectrum at room temperature. b Fluorescence emission spectra recorded at $77 \mathrm{~K}$

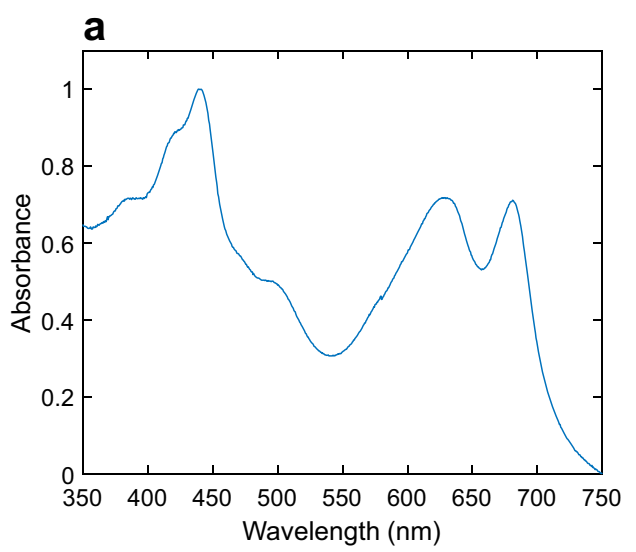

dependence of the spectra shows that fluorescence emission occurs before equilibration of the excitation energy between the PBS and the photosystems. The emission maximum upon $580 \mathrm{~nm}$ excitation is at $654 \mathrm{~nm}$, whereas the spectrum recorded with $460 \mathrm{~nm}$ excitation has a maximum at $684 \mathrm{~nm}$ and a broad PSI shoulder around $710 \mathrm{~nm}$.

\section{Time-resolved fluorescence spectroscopy}

We applied time-resolved fluorescence spectroscopy to resolve the dynamics of EET within the PBS, the photosystems and between them in intact Anabaena filaments. Measurements were performed with two excitation wavelengths, 580 or $460 \mathrm{~nm}$, predominantly exciting either PBS or Chls, respectively, and under conditions favouring open or closed PSII RCs. Representative DAES resulting from global lifetime analysis of the fluorescence recorded with $580 \mathrm{~nm}$ excitation are shown in Fig. 4. Minimum five exponential decay components were necessary for a good fit of the data (Supplementary Fig. S4). The resulting DAES have distinct shapes as they reflect emission from different groups of pigments (see the normalized DAES, Supplementary Fig. S5). The shortest resolved lifetimes are $28 \pm 4$ ps and $88 \pm 5$ ps (mean values \pm standard deviation from nine independent experiments). The first lifetime is characterized by fluorescence decay at $620-630 \mathrm{~nm}$ and rise at $660 \mathrm{~nm}$ indicated by the positive and negative peaks in the DAES, respectively (Fig. 4a). The second lifetime shows decay at $640 \mathrm{~nm}$ and rise at $670-680 \mathrm{~nm}$. The DAES strongly suggest EET from pigment pools emitting at the shorter wavelengths to acceptors emitting at the longer wavelengths. Therefore, we can conclude that the two components reflect EET between PC and APC pigments in the PBS. A decay component with a lifetime of $180 \pm 10$ ps dominates the kinetics in the 650-700 nm region; its maximum at $660 \mathrm{~nm}$ strongly suggests that it represents decay of excitations in APC. The $450 \pm 20$ ps decay lifetime, which has a relatively smaller contribution to the overall decay, can be ascribed to both PBS and PSII as it has peaks around $650-660 \mathrm{~nm}$ and $680 \mathrm{~nm}$ (Supplementary Fig. S5). The spectrum was found to vary somewhat between sample batches. Finally, a longlived component $(1.6 \pm 0.1 \mathrm{~ns})$ with emission maximum at $648 \mathrm{~nm}$ indicates a small (3\%) fraction of energetically uncoupled PC.

Closing the PSII RCs in the presence of DCMU and additional background illumination brought about changes
Fig. 4 DAES obtained from five-component global lifetime analysis of the fluorescence decays of intact Anabaena PCC 7120 filaments upon excitation at $580 \mathrm{~nm}$. a With open PSII RCs, $\mathbf{b}$ with closed PSII RCs
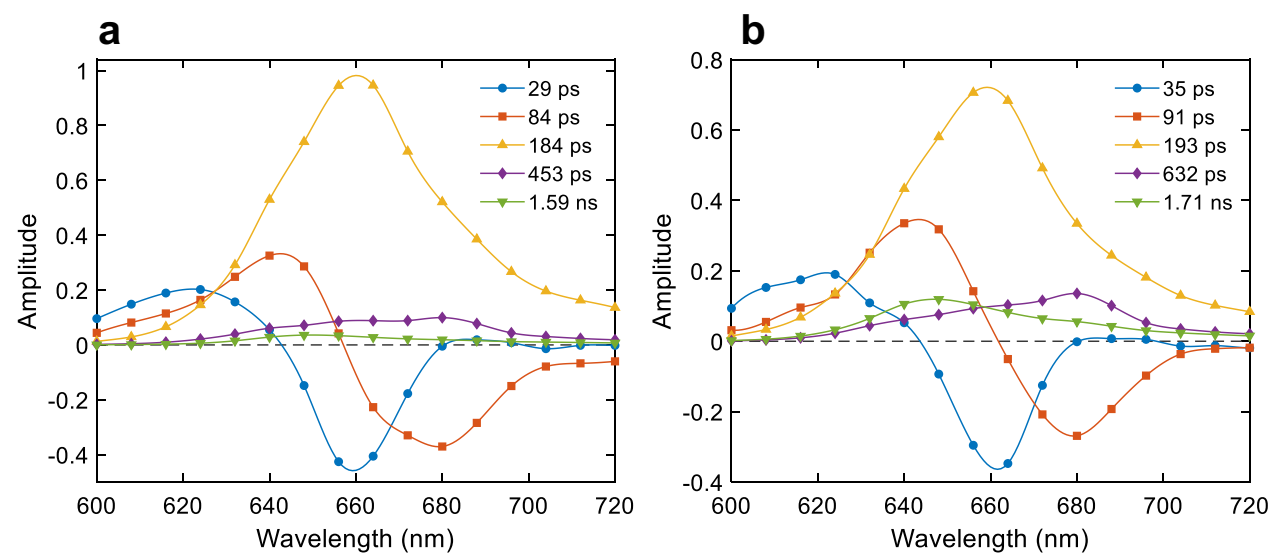
primarily in the three long-lived decay components (Fig. 4b). The 193 ps DAES consistently showed less emission at $680 \mathrm{~nm}$ (compare with the 184 ps DAES in open RCs). The fourth lifetime increased from $450 \pm 20$ to $590 \pm 20$ ps. Moreover, the relative amplitudes of the two longest-lived components increased significantly (see also Supplementary Fig. S6). Judging from the shape of the DAES, these components appear to originate from PSII as well as from the PBS.

The fluorescence kinetics upon $460 \mathrm{~nm}$ excitation is markedly different, largely because the initial excitations reside on the photosystems rather than the PBS antenna. Two lifetimes dominate the kinetics above $670 \mathrm{~nm}-10$ and $39 \mathrm{ps}$. The 10 ps lifetime was fixed-a marginally better numerical fit could be obtained with a value of 14 ps but with less realistic DAES (not shown). The first DAES (Fig. 5) shows fluorescence decay at $680 \mathrm{~nm}$ and rise at $720-740 \mathrm{~nm}$ and the second DAES shows overall decay with a maximum at $720 \mathrm{~nm}$. Based on the lifetimes and DAES, these components can be assigned to energy equilibration and trapping in PSI. The long-lived components $(195 \pm 20 \mathrm{ps}, 570 \pm 50 \mathrm{ps,}$ $1.7 \mathrm{~ns})$ have lifetimes and DAES similar to their respective

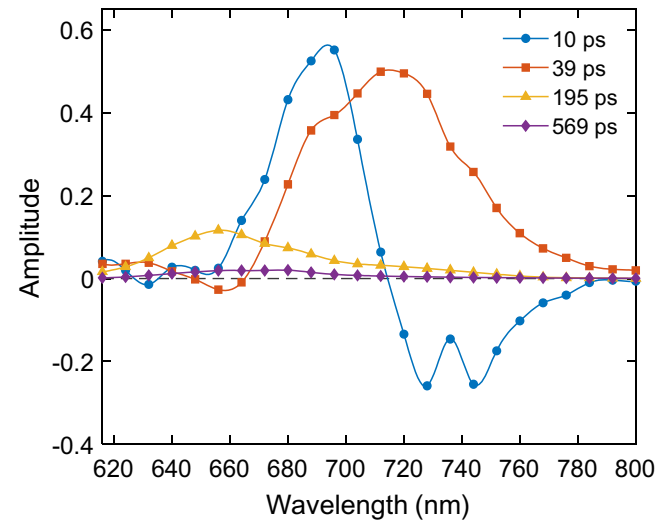

Fig. 5 DAES obtained from global lifetime analysis of the fluorescence decays of intact Anabaena PCC 7120 filaments upon excitation at $460 \mathrm{~nm}$ counterparts resolved upon $580 \mathrm{~nm}$ excitation but are of smaller relative amplitudes. A closer comparison of the long-lived DAES also reveals that all three components have higher relative amplitudes at $680 \mathrm{~nm}$ upon selective excitation of Chls-this was noticeable with either open or closed PSII RCs (Supplementary Fig. S6).

The excitation wavelength dependence of the fluorescence emission is also apparent in the stationary spectra, reconstructed from the DAES in " $F_{\mathrm{o}}$ " or " $F_{\mathrm{m}}$ " (i.e. preferentially open or closed PSII RCs, respectively), and in the calculated variable fluorescence spectra, $F_{\mathrm{v}}=F_{\mathrm{m}}-F_{\mathrm{o}}$ (Supplementary Fig. S7). Upon Chl excitation, the $F_{\mathrm{v}}$ spectrum peaks at $680 \mathrm{~nm}$ but lacks the distinct PSI-associated far-red emission band. Upon PBS excitation, there is a pronounced peak at $650 \mathrm{~nm}$, which is mainly due to the increased amplitude of the nanosecond decay component under " $F$ " conditions. The $F_{\mathrm{v}} / F_{\mathrm{m}}$ values of $0.3-0.4$, calculated from the timeresolved fluorescence, are lower than the typically observed values of 0.4-0.6 in dark-adapted cyanobacterial cultures (Campbell et al. 1998), possibly due to actinic effects of the measuring light and incomplete closure of the RCs.

To further disentangle the kinetics of EET in the photosynthetic complexes, we performed separate measurements on isolated PBS and isolated PSI. Four components were required for a satisfactory fit of the fluorescence of isolated PBS in the wavelength range $600-720 \mathrm{~nm}$ after $580 \mathrm{~nm}$ excitation (Fig. 6). The first two lifetimes (33 ps and 125 ps) have DAES closely reminiscent of the $29 \mathrm{ps}$ and 84 ps DAES resolved in intact filaments and representing EET within the PC rods and between the rods and the APC cores. The two longer lifetimes, $625 \mathrm{ps}$ and $1.7 \mathrm{~ns}$ have near-identical DAES shape with maxima at 645 and $660 \mathrm{~nm}$. The latter is the dominant decay component, whereas the faster component has a small amplitude. As a first approximation, the emission spectra of the main pigment pools can be obtained from a sequential irreversible kinetic model (Fig. 6b). The evolution-associated emission spectra (EAES) could be interpreted as three pigment pools: the first one emitting at
Fig. 6 Global lifetime analysis of the time-resolved fluorescence kinetics of isolated PBS with $580 \mathrm{~nm}$ excitation. a Decay-associated emission spectra, b evolution-associated emission spectra (normalized to the maximum)
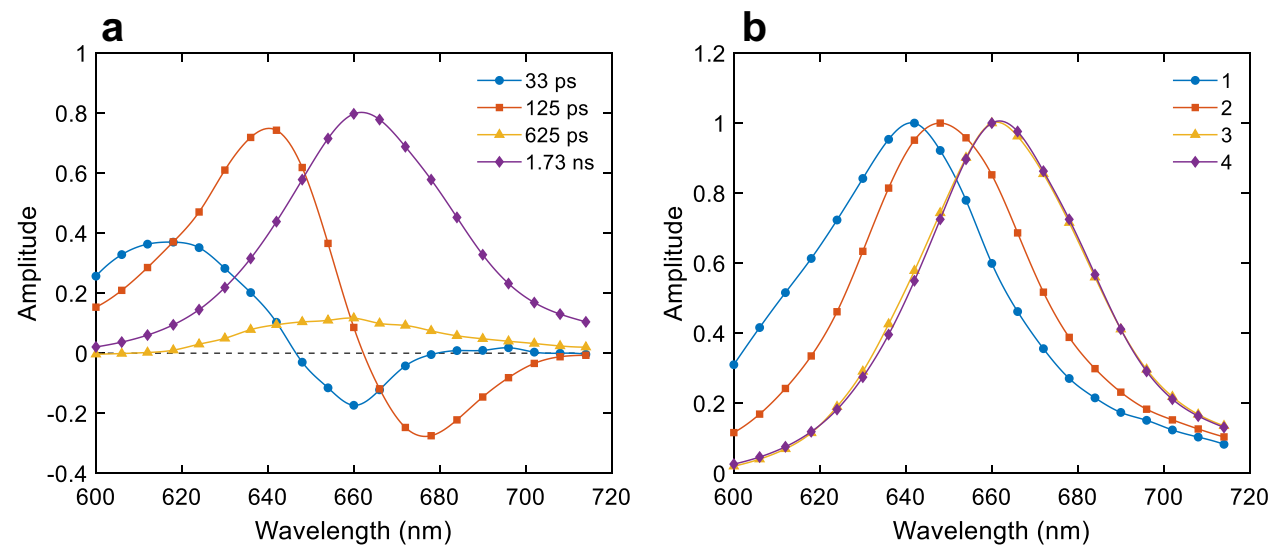
635-640 nm (PC) with a shoulder at 600-620 (PEC), the second emitting at 640-645 $\mathrm{nm}(\mathrm{PC})$, and the last at $660 \mathrm{~nm}$ with a shoulder at $680 \mathrm{~nm}$ (APC). Because the actual kinetic system is more complex, as discussed below, this is only a guiding assignment.

The fluorescence decay of PSI was followed by excitation at $460 \mathrm{~nm}$. DAES obtained from four-exponential global analysis are shown in Fig. 7. In close similarity to the fluorescence of intact cells excited at $460 \mathrm{~nm}$, two components with lifetimes of $10 \mathrm{ps}$ and $41 \mathrm{ps}$ dominate the kinetics. The $10 \mathrm{ps}$ DAES has a positive peak at $685 \mathrm{~nm}$ and a negative peak around $730 \mathrm{~nm}$, clearly representing energy equilibration between bulk PSI antenna Chls and low-energy "red" $\mathrm{Chl}$ forms. The $41 \mathrm{ps}$ DAES has only positive amplitude with a peak at $720 \mathrm{~nm}$ and a shoulder at $688 \mathrm{~nm}$, reflecting the trapping of equilibrated excitations in PSI (Fig. 7). Equilibration between the bulk and red forms is also observed in the raw time-resolved emission spectra as a shift of the maximum from 688 to $720 \mathrm{~nm}$ after 40 ps (Supplementary Fig. S8). Two long-lived components were required to fit the kinetics in a 4 ns window but their amplitudes are less than $1 \%$ of the main decay component. We hypothesize that these components originate from uncoupled pigments present in the sample; however, conclusive assignment cannot be made without detailed kinetic modelling analysis.

\section{Discussion}

Understanding the mechanisms and pathways of energy transfer in the cyanobacterial cells is far from trivial, given the large size of the PBS antenna, the variety of assemblies and structures that the PBP can accommodate, and the possibilities for vertical and lateral energy transfer between different PBPs in the same PBS or between PBS in densely packed arrays (Liu et al. 2008; Eyal et al. 2017; Harris et al. 2018).

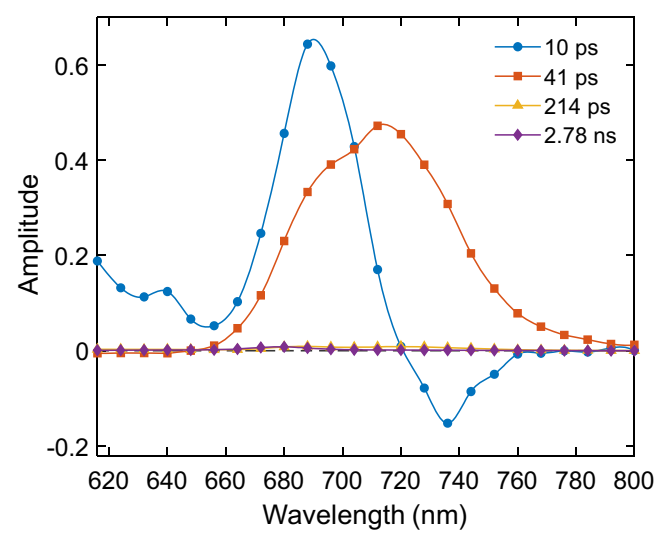

Fig. 7 DAES obtained from global analysis of the fluorescence kinetics of isolated PSI from Anabaena PCC 7120 recorded with excitation at $460 \mathrm{~nm}$
This complexity would account for an enormous number of kinetic parameters in modelling the excitation dynamics (Saer and Blankenship 2017), which are difficult or impossible to measure directly; hence, a complete energy transfer model of the entire system is lacking. The PBP composition and architecture of the PBS varies among species, which has impact on the dynamics and efficiency of light harvesting. With the present work we aimed to gain further insight into the dynamics of excitation energy transfer and trapping in Anabaena PCC 7120 by comparing the fluorescence kinetics in intact cells and in the isolated major pigment-protein complexes, PBS and PSI. Taken separately, the experimental results confirm a number of observations well documented in the literature and present some new findings as discussed below. As the fluorescence kinetics in vivo and in vitro were obtained under the same measurement conditions and from the same cell cultures, the combined results form an experimental basis that could be used for testing and comparing different kinetic models.

\section{Energy transfer in isolated PBS}

Global lifetime analysis of the fluorescence kinetics of isolated PBS resolved two decay lifetimes, 33 and $125 \mathrm{ps}$, that are clearly associated with energy transfer between PEC/ PC in the rods, APC, and the terminal emitters, APC-E and APC-D, of the PBS core. Based on the maxima of the EAES, it could be concluded that the 33 ps lifetime represents EET within the rods-from PVB in PEC and the $\beta-155$ PCB chromophores in PC to the lower-energy $\beta-84$ and $\alpha-84$ chromophores emitting at $640-650 \mathrm{~nm}$ (Debreczeny et al. 1993). Similarly, the 125 ps could be assigned to decay of $\mathrm{PC}$ and corresponding rise of emission from APC, i.e. to EET from the rods to the core. It is obvious from the DAES in Fig. 6 that the rise of the emission at wavelengths longer than $680 \mathrm{~nm}$ is described solely by the $125 \mathrm{ps}$ component, which is evidently the overall energy transfer time to the terminal emitters in the APC core. The shape of the final DAES indicates decay of equilibrated excitations among the APC emitting around $660 \mathrm{~nm}$ and the terminal emitters found in the basal core cylinders. Since we do not resolve the equilibration term, we can conclude that it is faster than the experimental time resolution-which is in agreement with earlier models, e.g. recent kinetic models of energy transfer in Synechocystis assigns APC660-APC680 "intradisk" equilibration time of about 3 ps (van Stokkum et al. 2018; Acuña et al. 2018a).

The decay lifetimes and their amplitudes at different wavelengths (DAES) are in good agreement with earlier reports on the EET of isolated PBS from various cyanobacteria. Suter et al. (1984) measured the fluorescence decay of PBS from Synechococcus sp. upon $580 \mathrm{~nm}$ excitation and found that the fluorescence at $590 \mathrm{~nm}$ decays primarily with 
a 25 ps lifetime, attributed to EET from the sensitizing to the fluorescing chromophores in PC. They also found that the $\mathrm{APC}$ emission at $680 \mathrm{~nm}$ rises over $130 \mathrm{ps}$, representing the effective time constant for rod-core transfer.

It should be noted that the effective time constants of EET represent a sum of a large number of microscopic rate constants and generally cannot be attributed to any individual hopping step between specific pigment groups. This is the reason why the effective rod-core transfer time depends on the length of the rods and can be as short as 17-18 ps in particles containing only one PC trimer (Sandström et al. 1988; Zhang et al. 1997). Thus, the observed results (DAES) can be interpreted differently depending on the choice of kinetic model. The DAES of Anabaena PBS shown here closely resemble the simulated DAES by Suter \& Holzwarth's (1987) kinetic model for the rod-core assembly except for the lack of a 10 ps EET lifetime. According to their model, the excitation migration along the rods is fast and the overall kinetics is limited by transfer to the APC core, which was considered as a single "trap" component. Tian et al. (2012) recorded the fluorescence kinetics of PBS from Synechocystis sp. using a streak camera and reported DAES that were also remarkably similar to the ones presented here, except for an additional rise lifetime of $8 \mathrm{ps}$. Although we have not been able to resolve such a component in our TCSPC experiments, it is conceivable that the true rise term might be even shorter than $8 \mathrm{ps}$ and beyond the experimental time resolution. In contrast to the earlier model by Suter and Holzwarth (1987), the 120 ps lifetime component was ascribed to EET within the APC core and equilibration between APC660 and the terminal emitters. This would seem a reasonable interpretation of the present results, considering that the 125 ps DAES has a negative maximum at $680 \mathrm{~nm}$ (Fig. 6). However, if we adopt a sequential kinetic model, the EAES show that the species being populated on a timescale of $33 \mathrm{ps}$ emits with a maximum at $650 \mathrm{~nm}$ (PC) and the $125 \mathrm{ps}$ lifetime populates a component with an emission maximum at $660 \mathrm{~nm}$ (APC). It can be concluded then that the former lifetime is associated with equilibration in the rods where the energy donors are PBV and blue-shifted (sensitizing) forms of PCB (e.g. the $\beta 155$ chromophores) and the acceptors are chromophores emitting around $650 \mathrm{~nm}$, presumably belonging to PC. This would fit the observed two spectral components in the $77 \mathrm{~K}$ emission spectra-at 640 and $650 \mathrm{~nm}$. Therefore, it appears more likely that the rate-limiting step in the kinetics is between the rods and the core, rather than spectral equilibration within the APC core. This does not mean, however, that EET in APC has no influence on the overall dynamics. All APC trimers contain blue-shifted and red-shifted PCB forms, absorbing around 610 and $650 \mathrm{~nm}$, respectively, with EET between them taking place in less than 1 ps to tens of ps (Holzwarth et al. 1990; Choubeh et al. 2018). Equilibration in APC trimers containing forms emitting at $680 \mathrm{~nm}$ (as in the basal ApcE and ApcF) has been shown to occur in 17 to 66 ps (Holzwarth et al. 1990; Zhao et al. 1998; Nganou et al. 2016). The effective lifetimes should be different in the fully assembled core. Therefore, both the 33 ps and the 125 ps lifetimes resolved in the intact Anabaena PBS probably involve pigments in PEC/PC as well as APC.

The additional decay lifetime in the range of $600-700 \mathrm{ps}$ is uncovered here likely thanks to a higher signal-to-noise ratio in the TCSPC data. As the spectra of this and the main decay component at $1.7 \mathrm{~ns}$ are very similar, they originate from the same pigment groups. It is possible that the $600 \mathrm{ps}$ decay reflects heterogeneity in the sample, like a subpopulation of mildly quenched APC. This quenching, however, is probably different from the photoprotective quenching of APC fluorescence induced by the orange carotenoid protein (OCP) (Kirilovsky and Kerfeld 2016), wherein a much higher deactivation rate is expected (Tian et al. 2011). The heterogeneity could thus be an artefact of the PBS isolation. However, it seems that it is an always present kinetic component, having approximately equal lifetime and relative amplitude in all tested preparations, and it is plausible that the same decay channel exists in vivo (see below). Alternatively, the decay lifetime may stem from excitation migration between subunits of the APC core, i.e. it is an intrinsic kinetic component. One could assume this to be specific feature of Anabaena due to the distinct architecture of the APC core. However, we could also resolve a similar 600-800 ps decay component in tri-cylindrical-core PBS from Synechocystis sp. (data not shown). The reason why it has not been reported earlier is most likely its relatively small amplitude.

\section{Photosystem I}

The fluorescence of isolated PSI in the picoseconds time range exhibits essentially biexponential fluorescence decay kinetics, which is typical for cyanobacteria (Gobets and van Grondelle 2001). Byrdin et al. (2000) reported fluorescence lifetimes of 13 and $37 \mathrm{ps}$ in PSI from T. elongatus with DAES very similar to the ones in Fig. 7 with one notable difference being the non-conservative shape of the 10 ps DAES. The positive and negative peaks in the DAES point to equilibration between the bulk antenna Chls in PSI emitting around $680 \mathrm{~nm}$ and the "red" Chls emitting at 710-720 nm. In Byrdin's (2000) experiments, the rise amplitude at $720 \mathrm{~nm}$ was significantly larger than the corresponding decay amplitude at $680 \mathrm{~nm}$, which was interpreted in terms of a kinetic model where excitations on the two pigment pools are trapped with different rate constants, assuming that trapping occurs before complete equilibration. Our results indicate that besides equilibration of bulk and red Chls there is already some trapping on the $10 \mathrm{ps}$ time scale 
(cf. Figs. 5 and 7). This will be verified by target analysis in a subsequent study. The 41 ps DAES shows the trapping of equilibrated excitations in the RC. This two-component kinetics of PSI is well resolved also in vivo upon $460 \mathrm{~nm}$ excitation-the two decay components of 10 and $39 \mathrm{ps}$ resolved in intact cells have spectra closely reminiscent of the corresponding DAES of isolated PSI.

As with other studies on cyanobacteria, we find that the great majority of Chls is connected with PSI and only a small fraction belongs to PSII. This is evident from the DAES of cells upon $460 \mathrm{~nm}$ excitation, which are dominated by the PSI-associated components (Fig. 5) as well as from the steady-state emission spectra recorded at $77 \mathrm{~K}$ (Fig. 3b).

\section{Energy transfer in intact cells}

The time-resolved fluorescence kinetics of intact Anabaena filaments share a lot of similarities with the reported kinetics from cells of different cyanobacterial strains (Mullineaux and Holzwarth 1991; Tian et al. 2011; Acuña et al. 2018b). Upon direct excitation of the PBS rods, the fluorescence kinetics in a few hundred picoseconds reflects primarily EET within the PBS, which is apparent by the similar DAES observed in cells and isolated PBS. The principal difference is that coupling of the PBS to photosystems in intact filaments shortens the APC decay lifetime to 170-200 ps as opposed to 1.5-1.7 ns in the isolated PBS. By global analysis of the fluorescence kinetics of WT Synechocystis cells, Tian et al (2011) observed three rise terms-at 645, 660 and $680 \mathrm{~nm}$, with lifetimes of 8,39 and $122 \mathrm{ps,} \mathrm{respec-}$ tively. They assigned the lifetimes to EET within the PC rods ( $8 \mathrm{ps})$, between the rods and the core ( $39 \mathrm{ps})$, and from APC660 to the core terminal emitters and Chls (122 ps). The latter two components bear obvious similarity with the 29 and 84 ps DAES of Anabaena filaments (Fig. 4) but also to the 33 and 125 ps DAES in isolated PBS (Fig. 6). As was described above, a simple sequential kinetic scheme of these data would suggest a different assignment of the intermediate species, i.e. rise of PC emitting at $650 \mathrm{~nm}$ on a $30 \mathrm{ps}$ timescale followed by transfer to APC (84-125 ps). This would fit with the PBS kinetics proposed by Holzwarth (Mullineaux and Holzwarth 1991; Suter and Holzwarth 1987) with rod-core equilibration occurring on 90-120 ps timescale in Synechococcus 6301. Interestingly, the PBSassociated lifetimes are consistently shorter in intact filaments than in isolated PBS. There could be several possible reasons for the observed differences. The decay lifetimes may be affected by EET between densely packed PBS (Harris et al. 2018) or between PBS and the photosystems. We cannot rule out the possibility of non-radiatively decaying $\mathrm{PC}$ in vitro, which would explain the non-conservative shape of the DAES in this case (Fig. 6a).
Tian's results showed fluorescence decay at 660-680 nm with a lifetime of 195 ps (again closely comparable to the 184 ps lifetime we find in Anabaena filaments), assigned to excitation trapping via charge separation in the PSII RCs. A more detailed kinetic model describing the same data included EET from PC to APC660 with an effective time constant of about 50 ps and from APC660 to APC680 of 35 ps. A similar analysis was applied to the kinetics of the CB mutant of Synechocystis (Tian et al. 2013), where the rods consist of only the proximal PC hexamer. The three rise terms, assigned to PC, APC660 and PSI/PSII in this case, had lifetimes of 7,25 and $77 \mathrm{ps.} \mathrm{Although} \mathrm{the} \mathrm{slowest}$ lifetime in this model is dominated by the coupling of APC and the photosystems, the rate-limiting step must still be equilibration within the PBS because practically the same lifetimes and DAES were observed in isolated PBS (Tian et al. 2012). This has been taken into account in the recent extended models of the kinetics of isolated PBS (van Stokkum et al. 2018) and PSI-deficient Synechocystis (Acuña et al. 2018a), wherein equilibration between cylinders in the PBS takes up to $200 \mathrm{ps}$ and the time constant of EET from the terminal emitters in the basal APC cylinders to Chls in PSII is $20 \mathrm{ps}$. The model is fundamentally different from the earlier analysis of Mullineaux and Holzwarth (1991), who interpret the 180-220 ps decay in Synechococcus 6301 cells as reflecting slow EET between APC and PSII, where charge separation occurs on a timescale of about 40 ps. However, the authors assumed that the emission originated from the terminal emitters, which is incompatible with the $660 \mathrm{~nm}$ emission maximum of the 184 ps DAES.

The longer-lived component (400-500 ps) can safely be attributed to electron transfer in PSII, owing to its maximum at $680 \mathrm{~nm}$. However, also in this case, the observed secondary peaks in the $640-660 \mathrm{~nm}$ region indicate that even this component is not associated with PSII only but also reflects decay of excitations in the PBS (Supplementary Fig. S5). We therefore propose that the $600 \mathrm{ps}$ fluorescence decay lifetime in isolated PBS represents an intrinsic component of the antenna kinetics that is also observed in vivo. In agreement with this, we notice that the amplitude of the 500 ps component in cells is diminished at $640-660 \mathrm{~nm}$ relative to $680 \mathrm{~nm}$ upon predominant excitation of Chls at $460 \mathrm{~nm}$ (Fig. 5, Supplementary Fig. S5). On the other hand, the amplitude at $680 \mathrm{~nm}$ increases upon Chl excitation relative to the $200 \mathrm{ps}$ component (APC), corroborating that the emission at this wavelength originates from Chls.

As discussed above, there are three different interpretations in the literature regarding the main APC decay time of 180 ps reported here for Anabaena filaments: equilibration in the PBS, EET from APC to PSII and trapping in PSII. These three scenarios can be referred to as migration-limited, transfer-to-trap-limited and trap-limited, respectively. We can argue against a pure trap-limited scenario, because 
trapping in the isolated PSII core occurs with a main lifetime of 30-50 ps (Holzwarth et al. 2006), i.e. significantly faster than the observed decay upon PBS excitation. The lifetime must then reflect energy equilibration in the PBS-PSII supercomplex. Furthermore, if we assume rapid and nearly irreversible EET from APC to the PSII antenna, then APC should be depopulated faster, whereas the data show slowly decaying emission at $660 \mathrm{~nm}$. On the same grounds we can exclude the transfer-to-trap-limited case where the single rate-limiting step is coupling of the terminal emitters (APC680) to Chls in PSII or PSI-the bulk APC660 would be depopulated faster than APC680. In principle, the fluorescence kinetics of isolated PBS and of intact cells with open PSII RCs could be interpreted in the frame of a model where APC660 and APC680 equilibrate rapidly and the rate-limiting step is between APC and Chls (Mullineaux and Holzwarth 1991).

\section{Variable PSII fluorescence}

The contribution of PSII charge separation to the overall excitation dynamics in cells can be inferred by comparing the fluorescence kinetics recorded with open and closed PSII RCs (Mullineaux and Holzwarth 1993, 1991; Remelli and Santabarbara 2018; Santabarbara et al. 2019). Our results well reproduced what has been reported previously for Synechocystis-in " $F_{\mathrm{m}}$ " conditions, the short-lived decay components mainly associated with the PBS were barely affected, the main decay component lifetime (160-190 ps) remained virtually the same, whereas the $\sim 400$ ps lifetime increased along with its relative amplitude. The invariance of the 160-190 ps lifetime rules out purely trap-limited dynamics. However, in our experiments the shape of the $180-190$ ps DAES varies-in $F_{\mathrm{m}}$ conditions, its amplitude at $680 \mathrm{~nm}$ is higher, suggesting decay of PSII antenna states and not only APC. This leads to a model where APC680-Chl transfer is fast and the kinetics is determined by a complex energy migration in the PBS (van Stokkum et al. 2018). Then again, it is surprising that the overall kinetics is virtually the same in species like Anabaena and Synechocystis having different organizations of the PBS and varying in the number and size of PBPs in the rods and the core. Ultimately, target analysis based on simultaneous model fitting of the isolated complexes and intact filaments at different excitation conditions and in different states of PSII should help distinguish between these scenarios.

Interestingly, the longest-lived fluorescence component $(1.5-1.7 \mathrm{~ns})$ increases its contribution under $F_{\mathrm{m}}$ conditions (with DCMU and additional background illumination). While the long-lived emission at $680 \mathrm{~nm}$ has been earlier observed (Remelli and Santabarbara 2018) and attributed to the closed PSII RCs, we also found increased relative amplitudes at $640 \mathrm{~nm}$, i.e. from PC (Supplementary Fig.
S6). In $F_{\mathrm{o}}$ conditions, this component originates from around $3 \%$ of uncoupled PC rods, but the number increased to $10 \%$ when the sample was continuously illuminated. Because of the long lifetime, the component has the dominant contribution to the stationary fluorescence at $640-660 \mathrm{~nm}$ in $F_{\mathrm{m}}$ conditions and, consequently, to the variable fluorescence spectrum and the $F_{\mathrm{v}} / F_{\mathrm{m}}$ spectrum (Supplementary Fig. S7). The variable fluorescence presumably originates only from PSII and its associated antenna. If this is true, it would follow that the emission at $650 \mathrm{~nm}$ is a result of energy equilibration between PSII and the PBS. However, the excitation dependence of the variable fluorescence is an evidence against such an interpretation. As pointed out by Remelli and Santabarbara (2018), we can expect the $F_{\mathrm{v}}$ spectrum to be independent from excitation wavelength, if there is rapid equilibration between the two systems (PBS and PSII). This has indeed shown to be the case in different cyanobacterial species (Remelli and Santabarbara 2018; Santabarbara et al. 2019). In contrast, in our experiments the $640 \mathrm{~nm}$ peak in the $F_{\mathrm{v}}$ spectrum is observed upon preferential PBS excitation at $580 \mathrm{~nm}$ (Supplementary Fig. S7). Moreover, slow back transfer to the PBS would incur a rise term at $640 \mathrm{~nm}$ upon Chls excitation, which is not observed. Therefore, it seems more likely that the cause of this emission is PBS or PBS rods that are uncoupled from PSII in $F_{\mathrm{m}}$ conditions. This may be a consequence of the measurement protocol using a combination of DCMU and prolonged background illumination and related to the previously reported light-induced detachment of PBS (Chukhutsina et al. 2015; Tamary et al. 2012; Stoitchkova et al. 2007). This phenomenon will be investigated further.

\section{Conclusions}

The kinetics of EET in the PBS of Anabaena PC 7120 was found to be remarkably similar to other cyanobacterial PBS, notably that of Synechocystis. This is somewhat surprising considering the differences in composition and architecture of the two systems. The effective rod-core equilibration time was found to be about 125 ps and equilibration with the terminal emitters in APC was faster than the experiment time resolution.

The picosecond excited state dynamics of isolated tetrameric PSI from Anabaena can be described with two lifetimes of about $10 \mathrm{ps}$ and $40 \mathrm{ps}$ that represent energy equilibration with low-energy "red" Chl forms and trapping of the equilibrated excitations, respectively. The slower trapping compared to other cyanobacterial species might be related to the higher number and lower-energy of the "red" Chls in Anabaena. Because the number of Chls associated with PSI exceeds fivefold the Chls in PSII, the fluorescence kinetics of intact cells is dominated by PSI, allowing us to resolve 
both the equilibration with the red Chls and trapping kinetics in PSI in vivo.

In intact filaments, virtually all energy absorbed by the PBS was rapidly transferred to the photosystems. We found about $3 \%$ of PC uncoupled from the rest of the system, which, however contributed to $22 \%$ of the fluorescence emission at $650 \mathrm{~nm}$. No uncoupled PEC was detected. EET within the PBS is well resolved in the intact filaments and trapping of PBS excitations occurs on a timescale of 170-200 ps, similar to previous reports. The lifetime is most likely determined by EET within the PBS (primarily the APC core) and between the PBS and the membrane complexes. The inverted kinetics regime, where EET to the photosystems is effectively slower than trapping by charge separation, makes it very difficult to directly resolve the two photosystems upon PBS excitation, as the Chl excited states are depopulated faster via charge separation than they are populated by the PBS. In principle, simultaneous model fitting of the results obtained with selective excitation of PBS and Chls could resolve this ambiguity; however, this is by far not trivial, as one must consider the coexistence of an a priori unknown number of PSI and PSII that have or have no contact with PBS (van Stokkum et al. 2018). Kinetic modelling of this kind, performed with the data reported here, will be presented in a subsequent work.

Acknowledgements Open access funding provided by Biological Research Centre, Szeged. The authors thank Terézia Kovács, Sindhujaa Vajravel and Fanny Kata Balog-Vig for help with the sample preparations. The work was supported by grants from the Hungarian Ministry for National Economy (GINOP-2.3.2-15-2016-00001) and the National Research, Development and Innovation Office (NKFIH NN-124904; 2018-1.2.1-NKP-2018-00009). A. B. was supported by the European Union's Horizon 2020 research and innovation program under the Marie Skłodowska-Curie Grant Agreement No 675006 (SE2B).

Funding Open access funding provided by MTA Biological Research Center (MTA SZBK).

Data availability The time-resolved fluorescence data reported here are deposited at Mendeley Data (Akhtar and Lambrev 2019).

\section{Compliance with ethical standards}

Conflict of interest The authors declare that they have no competing interest.

Open Access This article is licensed under a Creative Commons Attribution 4.0 International License, which permits use, sharing, adaptation, distribution and reproduction in any medium or format, as long as you give appropriate credit to the original author(s) and the source, provide a link to the Creative Commons licence, and indicate if changes were made. The images or other third party material in this article are included in the article's Creative Commons licence, unless indicated otherwise in a credit line to the material. If material is not included in the article's Creative Commons licence and your intended use is not permitted by statutory regulation or exceeds the permitted use, you will need to obtain permission directly from the copyright holder. To view a copy of this licence, visit http://creativecommons.org/licenses/by/4.0/.

\section{References}

Acuña A, Van Alphen P, Van Grondelle R, Van Stokkum I (2018a) The phycobilisome terminal emitter transfers its energy with a rate of (20 ps)-1 to photosystem II. Photosynthetica 56(1):265-274

Acuña AM, Lemaire C, van Grondelle R, Robert B, van Stokkum IH (2018b) Energy transfer and trapping in Synechococcus WH 7803. Photosynth Res 135(1-3):115-124

Akhtar P, Lambrev PH (2019) Time-resolved fluorescence data of Anabaena PCC 7120. Mendeley Data V1. https://doi.org/10.17632 /89zfxfkrp3.1

Akhtar P, Lingvay M, Kiss T, Deák R, Bóta A, Ughy B, Garab G, Lambrev PH (2016) Excitation energy transfer between light-harvesting complex II and photosystem I in reconstituted membranes. Biochim Biophys Acta 4:462-472

Andrizhiyevskaya EG, Schwabe TME, Germano M, D'Haene S, Kruip J, van Grondelle R, Dekker JP (2002) Spectroscopic properties of PSI-IsiA supercomplexes from the cyanobacterium Synechococcus PCC 7942. Biochim Biophys Acta 1556(2-3):265-272

Arteni AA, Ajlani G, Boekema EJ (2009) Structural organisation of phycobilisomes from Synechocystis sp. strain PCC6803 and their interaction with the membrane. Biochim Biophys Acta 1787(4):272-279

Bar-Eyal L, Shperberg-Avni A, Paltiel Y, Keren N, Adir N (2018) Light harvesting in cyanobacteria: the phycobilisomes. In: Croce R, Van Grondelle R, Van Amerongen H, Van Stokkum IHM (eds) Light harvesting in photosynthesis. Foundations of biochemistry and biophysics, 1st edn. CRC Press, Boca Raton, pp 77-93

Bittersmann E, Holzwarth AR, Agel G, Nultsch W (1988) Picosecond time-resolved emission spectra of photoinhibited and photobleached Anabaena variabilis. Photochem Photobiol 47(1):101-105

Byrdin M, Rimke I, Schlodder E, Stehlik D, Roelofs TA (2000) Decay kinetics and quantum yields of fluorescence in photosystem I from Synechococcus elongatus with $\mathrm{P}_{700}$ in the reduced and oxidized state: are the kinetics of excited state decay trap-limited or transfer-limited? Biophys J 79(2):992-1007

Campbell D, Hurry V, Clarke AK, Gustafsson P, Öquist G (1998) Chlorophyll fluorescence analysis of cyanobacterial photosynthesis and acclimation. Microbiol Mol Biol Rev 62(3):667-683

Choubeh RR, Sonani RR, Madamwar D, Struik PC, Bader AN, Robert $B$, van Amerongen H (2018) Picosecond excitation energy transfer of allophycocyanin studied in solution and in crystals. Photosynth Res 135(1-3):79-86

Chukhutsina V, Bersanini L, Aro E-M, Van Amerongen H (2015) Cyanobacterial light-harvesting phycobilisomes uncouple from photosystem I during dark-to-light transitions. Sci Rep 5:14193

Debreczeny MP, Sauer K, Zhou J, Bryant DA (1993) Monomeric C-phycocyanin at room temperature and $77 \mathrm{~K}$ : resolution of the absorption and fluorescence spectra of the individual chromophores and the energy-transfer rate constants. J Phys Chem 97(38):9852-9862

Ducret A, Müller SA, Goldie KN, Hefti A, Sidler WA, Zuber H, Engel A (1998) Reconstitution, characterisation and mass analysis of the pentacylindrical allophycocyanin core complex from the cyanobacterium Anabaena sp. PCC 71201. J Mol Biol 278(2):369-388

El-Mohsnawy E, Kopczak MJ, Schlodder E, Nowaczyk M, Meyer HE, Warscheid B, Karapetyan NV, Rögner M (2010) Structure and function of intact photosystem 1 monomers from the cyanobacterium Thermosynechococcus elongatus. Biochemistry 49(23):4740-4751

Eyal LB, Choubeh RR, Cohen E, Eisenberg I, Tamburu C, Dorogi M, Ünnep R, Appavou M-S, Nevo R, Raviv U (2017) Changes in aggregation states of light-harvesting complexes as a mechanism 
for modulating energy transfer in desert crust cyanobacteria. Proc Natl Acad Sci USA 114(35):9481-9486

Garnier F, Dubacq J-P, Thomas J-C (1994) Evidence for a transient association of new proteins with the Spirulina maxima phycobilisome in relation to light intensity. Plant Physiol 106(2):747-754

Glauser M, Bryant DA, Frank G, Wehrli E, Rusconi SS, Sidler W, Zuber H (1992) Phycobilisome structure in the cyanobacteria Mastigocladus laminosus and Anabaena sp. PCC 7120. Eur J Biochem 205(3):907-915

Glazer AN (1984) Phycobilisome a macromolecular complex optimized for light energy transfer. Biochim Biophys Acta 768(1):29-51

Gobets B, van Grondelle R (2001) Energy transfer and trapping in photosystem I. Biochim Biophys Acta 1507(1-3):80-99

Gobets B, van Stokkum IHM, Rogner M, Kruip J, Schlodder E, Karapetyan NV, Dekker JP, van Grondelle R (2001) Time-resolved fluorescence emission measurements of photosystem I particles of various cyanobacteria: a unified compartmental model. Biophys J 81(1):407-424

Harris D, Bar-Zvi S, Lahav A, Goldshmid I, Adir N (2018) The structural basis for the extraordinary energy-transfer capabilities of the phycobilisome. In: Harris JR, Boekema EJ (eds) Membrane protein complexes: structure and function subcellular biochemistry, vol 87. Springer, New York, pp 57-82

Holzwarth AR (1991) Structure-function relationships and energy transfer in phycobiliprotein antennae. Physiol Plant 83(3):518-528

Holzwarth AR, Bittersmann E, Reuter W, Wehrmeyer W (1990) Studies on chromophore coupling in isolated phycobiliproteins: III. Picosecond excited state kinetics and time-resolved fluorescence spectra of different allophycocyanins from Mastigocladus laminosus. Biophys J 57(1):133-145

Holzwarth AR, Muller MG, Reus M, Nowaczyk M, Sander J, Rogner M (2006) Kinetics and mechanism of electron transfer in intact photosystem II and in the isolated reaction center: pheophytin is the primary electron acceptor. Proc Natl Acad Sci USA 103(18):6895-6900

Jallet D, Thurotte A, Leverenz RL, Perreau F, Kerfeld CA, Kirilovsky D (2014) Specificity of the cyanobacterial orange carotenoid protein: influences of orange carotenoid protein and phycobilisome structures. Plant Physiol 164(2):790-804

Karapetyan NV, Schlodder E, van Grondelle R, Dekker JP (2006) The long-wavelength chlorophylls of photosystem I. In: Golbeck JH (ed) Photosystem I: the light-driven, plastocyanin: ferredoxin oxidoreductase advances in photosynthesis and respiration, vol 24. Springer, Dordrecht, pp 177-192

Kato K, Nagao R, Jiang T-Y, Ueno Y, Yokono M, Chan SK, Watanabe M, Ikeuchi M, Shen J-R, Akimoto S (2019) Structure of a cyanobacterial photosystem I tetramer revealed by cryo-electron microscopy. Nat Commun 10(1):1-9

Kirilovsky D, Kerfeld CA (2016) Cyanobacterial photoprotection by the orange carotenoid protein. Nat Plants 2(12):16180

Kondo K, Ochiai Y, Katayama M, Ikeuchi M (2007) The membraneassociated CpcG2-phycobilisome in Synechocystis: a new photosystem I antenna. Plant Physiol 144(2):1200-1210

Liu L-N, Aartsma TJ, Thomas J-C, Lamers GE, Zhou B-C, Zhang Y-Z (2008) Watching the native supramolecular architecture of photosynthetic membrane in red algae topography of phycobilisomes and their crowding, diverse distribution patterns. J Biol Chem 283(50):34946-34953

Liu H, Zhang H, Niedzwiedzki DM, Prado M, He G, Gross ML, Blankenship RE (2013) Phycobilisomes supply excitations to both photosystems in a megacomplex in cyanobacteria. Science 342(6162):1104-1107

Liu H, Weisz DA, Zhang MM, Cheng M, Zhang B, Zhang H, Gerstenecker GS, Pakrasi HB, Gross ML, Blankenship RE
(2019) Phycobilisomes harbor FNRL in cyanobacteria. mBio 10(2):e00619-e00669

Lundell DJ, Glazer AN, Melis A, Malkin R (1985) Characterization of a cyanobacterial photosystem I complex. J Biol Chem 260:646-654

MacColl R (2004) Allophycocyanin and energy transfer. Biochim Biophys Acta 1657(2-3):73-81

Mullineaux CW (1994) Excitation energy transfer from phycobilisomes to photosystem I in a cyanobacterial mutant lacking photosystem II. Biochim Biophys Acta 1184(1):71-77

Mullineaux CW, Holzwarth AR (1991) Kinetics of excitation energy transfer in the cyanobacterial phycobilisome-photosystem II complex. Biochim Biophys Acta 1098(1):68-78

Mullineaux CW, Holzwarth AR (1993) Effect of photosystem II reaction centre closure on fluorescence decay kinetics in a cyanobacterium. Biochim Biophys Acta 1183(2):345-351

Nganou C, David L, Adir N, Mkandawire M (2016) Linker proteins enable ultrafast excitation energy transfer in the phycobilisome antenna system of Thermosynechococcus vulcanus. Photochem Photobiol Sci 15(1):31-44

Niedzwiedzki DM, Liu H, Blankenship RE (2019) Excitation energy transfer in intact CpcL-phycobilisomes from Synechocystis sp. PCC 6803. J Phys Chem B 123(22):4695-4704

Nultsch W, Bittersmann E, Holzwarth AR, Agel G (1990) Effects of strong light irradiation on antennae and reaction centres of the cyanobacterium Anabaena variabilis: a time-resolved fluorescence study. J Photochem Photobiol B 5(3):481-494

Ogawa T, Vernon LP, Yamamoto HY (1970) Properties of Anabaena variabilis cells grown in the presence of diphenylamine. Biochim Biophys Acta 197(2):302-307

Peterson RB, Dolan E, Calvert HE, Ke B (1981) Energy transfer from phycobiliproteins to photosystem I in vegetative cells and heterocysts of Anabaena variabilis. Biochim Biophys Acta 634:237-248

Remelli W (1859) Santabarbara S (2018) Excitation and emission wavelength dependence of fluorescence spectra in whole cells of the cyanobacterium Synechocystis sp. PPC6803: influence on the estimation of photosystem II maximal quantum efficiency. Biochim Biophys Acta 11:1207-1222

Rippka R, Deruelles J, Waterbury JB, Herdman M, Stanier RY (1979) Generic assignments, strain histories and properties of pure cultures of cyanobacteria. Microbiology 111(1):1-61

Rögner M, Nixon PJ, Diner BA (1990) Purification and characterization of photosystem I and photosystem II core complexes from wild-type and phycocyanin-deficient strains of the cyanobacterium Synechocystis PCC 6803. J Biol Chem 265(11):6189-6196

Saer RG, Blankenship RE (2017) Light harvesting in phototrophic bacteria: structure and function. Biochem J 474(13):2107-2131

Sandström Å, Gillbro T, Sundström V, Wendler J, Holzwarth AR (1988) Picosecond study of energy transfer within 18-S particles of AN 112 (a mutant of Synechococcus 6301) phycobilisomes. Biochim Biophys Acta 933(1):54-64

Santabarbara S, Villafiorita Monteleone F, Remelli W, Rizzo F, Menin B, Casazza AP (2019) Comparative excitation-emission dependence of the $\mathrm{F}_{\mathrm{V}} / \mathrm{F}_{\mathrm{M}}$ ratio in model green algae and cyanobacterial strains. Physiol Plant 166(1):351-364

Scott M, McCollum C, Vasilev S, Crozier C, Espie GS, Krol M, Huner NP, Bruce D (2006) Mechanism of the down regulation of photosynthesis by blue light in the cyanobacterium Synechocystis sp. PCC 6803. Biochemistry 45(29):8952-8958

Stoitchkova K, Zsiros O, Jávorfi T, Páli T, Andreeva A, Gombos Z, Garab G (2007) Heat- and light-induced reorganizations in the phycobilisome antenna of Synechocystis sp. PCC 6803. Thermooptic effect. Biochim Biophys Acta 1767(6):750-756

Suter GW, Holzwarth AR (1987) A kinetic model for the energy transfer in phycobilisomes. Biophys J 52(5):673-683 
Suter GW, Mazzola P, Wendler J, Holzwarth AR (1984) Fluorescence decay kinetics in phycobilisomes isolated from the bluegreen alga Synechococcus 6301. Biochim Biophys Acta 766(2):269-276

Switalski SC, Sauer K (1984) Energy transfer among the chromophores of C-phycocyanin from Anabaena variabilis using steady state and time-resolved fluorescence spectroscopy. Photochem Photobiol 40(4):423-427

Tamary E, Kiss V, Nevo R, Adam Z, Bernat G, Rexroth S, Rogner M, Reich Z (2012) Structural and functional alterations of cyanobacterial phycobilisomes induced by high-light stress. Biochim Biophys Acta 2:319-327

Tian L, van Stokkum IH, Koehorst RB, Jongerius A, Kirilovsky D, van Amerongen H (2011) Site, rate, and mechanism of photoprotective quenching in cyanobacteria. J Am Chem Soc 133(45):18304-18311

Tian L, Gwizdala M, van Stokkum IH, Koehorst RB, Kirilovsky D, van Amerongen $\mathrm{H}$ (2012) Picosecond kinetics of light harvesting and photoprotective quenching in wild-type and mutant phycobilisomes isolated from the cyanobacterium Synechocystis PCC 6803. Biophys J 102(7):1692-1700

Tian L, van Stokkum IH, Koehorst RB, van Amerongen H (2013) Light harvesting and blue-green light induced non-photochemical quenching in two different C-phycocyanin mutants of Synechocystis PCC 6803. J Phys Chem B 117(38):11000-11006

Vajravel S, Kis M, Kłodawska K, Laczko-Dobos H, Malec P, Kovács L, Gombos Z, Toth T (2017) Zeaxanthin and echinenone modify the structure of photosystem I trimer in Synechocystis sp. PCC 6803. Biochim Biophys Acta 7:510-518

van Stokkum IH, Gwizdala M, Tian L, Snellenburg JJ, van Grondelle $\mathrm{R}$, van Amerongen H, Berera R (2018) A functional compartmental model of the Synechocystis PCC 6803 phycobilisome. Photosynth Res 135(1-3):87-102

Watanabe M, Semchonok DA, Webber-Birungi MT, Ehira S, Kondo K, Narikawa R, Ohmori M, Boekema EJ, Ikeuchi M (2014) Attachment of phycobilisomes in an antenna-photosystem I supercomplex of cyanobacteria. Proc Natl Acad Sci USA 111(7):2512-2517

Zhang J-M, Zhao J-Q, Jiang L-J, Zheng X-G, Zhao F-L, Wang H-Z (1997) Studies on the energy transfer among the rod-core complex from phycobilisome of Anabaena variabilis by time resolved fluorescence emission and anisotropy spectra. Biochim Biophys Acta 1320(3):285-296

Zhao F, Zheng X, Zhang J, Wang H, Yu Z, Zhao J, Jiang L (1998) Energy transfer in allophycocyanin hexamer from Anabaena variabilis by time-resolved spectroscopy. J Photochem Photobiol B 45(2-3):144-149

Publisher's Note Springer Nature remains neutral with regard to jurisdictional claims in published maps and institutional affiliations. 